\title{
UN PRUSSIANO A ROMA: LE LETTERE ITALIANE DI WINCKELMANN
}

\author{
GABRIELLA CATALANO (*)
}

SunTO. - Nell'epistolario plurilingue di Johann Joachim Winckelmann, l'italiano asseconda la finalità di esporre la propria adesione al contesto straniero di accoglienza. $\mathrm{Ma}$, al di là di un uso pragmatico, Winckelmann avverte la vitalità del nuovo idioma che acquisisce non senza difficoltà e imperfezioni. Anche grazie alle interferenze dal tedesco la lingua italiana, sperimentata nelle lettere, si dimostra in linea con la libertà stilistica e la vis polemica del fondatore della storia dell'arte.

$* * *$

ABSTRACT. - In the multilingual epistolary by JJW, writing in Italian goes along with the aim of showing his agreement to the foreign welcome context/background. However, beyond its pragmatic function, W feels the energy and enthusiasm of the new idiom that he learns not without any difficulties and imperfections. Thanks to the interferences from German, the Italian language experienced in his letters, is in line with the stylistic freedom and the vis polemica of the founder of the history of art.

Basterebbe solo sfogliare i quattro volumi dell'epistolario di Johann Joachim Winckelmann, editi negli anni Cinquanta del secolo scorso da Walther Rehm, per rendersi conto della varietà di idiomi che connota una corrispondenza folta, diseguale, ricchissima di ogni genere di notizie e riflessioni. ${ }^{1}$ Nell'alternanza di finalità e di toni che caratte-

(*) Università di Roma Tor Vergata, Roma, Italia.

E-mail: gabriella.catalano99@gmail.com

1 All'edizione tedesca delle lettere J.J. Winckelmann, Briefe, ed. storico-critica a cura di W. Rehm, De Gruyter, Berlin 1952-1957, voll. 4 e all'edizione delle Lettere italiane, a cura di G. Zampa, Milano 1961 si è aggiunta di recente un'edizione italiana completa dell'epistolario in tre volumi, promossa dall'Istituto di Studi Germanici: J.J. Winckelmann, Lettere, a cura di M. Fancelli e J. Raspi Serra, coordinamento scientifico di F. Cambi, Roma 2016. D'ora in poi questa edizione sarà citata con l'abbreviazione 
rizza le lettere in cui il fondatore dell'archeologia moderna interagisce con amici e conoscenti, dà libero sfogo ai propri sentimenti, tenta manovre diplomatiche e porta avanti veementi polemiche contro esperti d'arte e di antichità, la diversità linguistica contribuisce ad accrescere la costante impressione di mobilità: Winckelmann scrive in latino, italiano, francese, tedesco, alterna differenti lingue in lettere allo stesso destinatario, anche all'interno della stessa lettera. Al conte Heinrich von Bünau, presso il quale lavorò a Nöthnitz nei primi anni trascorsi a Dresda, Winckelmann scrive sia in tedesco sia in latino; al bolognese Gian Ludovico Bianconi, medico di corte e letterato, sia in francese che in italiano; e rivolgendosi al filologo Christian Gottlob Heyne adotta nel corso di un'unica lettera prima il tedesco e poi l'italiano non tralasciando di esporre, di fronte a quel competentissimo esperto di antichità, la propria dimestichezza nelle lingue classiche con un'ampia citazione dal greco tradotta in latino. Il divertissement linguistico sotteso a tale plurilinguismo è congeniale alla volontà di autostilizzazione che porta Winckelmann a modulare in base al destinatario la propria genealogia culturale. ${ }^{2}$ L'interazione epistolare lo vede intento a esibire le conoscenze acquisite o a favorire un'immagine di sé che può tornare utile per ottenere commissioni e nomine attestando l'avvenuto radicamento in terra italiana, dove si era trasferito dal 1755. Si tratta di ragioni pragmatiche, dovute al destinatario come al mittente, che guidano anche la scelta della lingua italiana nella corrispondenza, ritenuta più vicina alle lingue classiche e quindi maggiormente adatta a ricreare quella repubblica dei dotti a cui lo studioso tedesco aspirava nella sua utopia di un ritorno al classico: è probabilmente in questo senso che va interpretato l'uso della lingua italiana anche nelle lettere ai connazionali, come l'amico pittore Raphael Mengs o il cognato di questi, Anton Maron.

La corrispondenza italiana rappresenta, all'interno dell'epistolario di Winckelmann (841 lettere), un piccolo nucleo di 125 lettere,

Lett. a cui seguirà l'indicazione del volume in carattere romano e della pagina in cifra araba. Per la storia delle edizioni delle lettere si rimanda al saggio introduttivo di M. Fancelli, Passione per l'antico e vita: per una storia delle edizioni delle lettere, nel primo volume di questa edizione, pp. 39-65.

2 Alla scrittura epistolare come strumento di autorappresentazione rinvia M. Disselkamp, Die Stadt der Gelebrten. Studien zu Johann Joachim Winckelmanns Briefen aus Rom, Tübingen 1993, p. 21. 
essenziali tuttavia per comprendere il valore attribuito all'uso della lingua straniera all'interno di un rapporto che non fu privo di contraddizioni e conflitti. ${ }^{3}$ Se la conoscenza della lingua italiana rivela un costante desiderio di integrazione, la sua acquisizione così come la sua pratica vanno analizzati in un contesto multilingue. Una varietà che corrisponde pienamente a una vocazione fondamentale del Settecento, il "secolo d'oro della Interlinguistica", come lo ha definito Gianfranco Folena. ${ }^{4}$ In quell'epoca schiettamente europea la lingua tedesca, purismi permettendo, andava conquistando lentamente il proprio prestigio culturale. Sebbene nel dizionario dei fratelli Grimm - che Jacob, il più anziano dei due, compilò fino alla lettera F e alla parola Furcht - vengano con cura estromessi moltissimi forestierismi, anche derivanti dal latino, il tedesco moderno si costruiva in un dialogo costante con le altre lingue. ${ }^{5}$ Tanto che August Wilhelm Schlegel, nel saggio su Ludwig Tieck traduttore pubblicato nelle pagine dell'Athenaeum, ne volle celebrare la flessibilità nella capacità di assimilazione degli idiomi stranieri, espressione di una vera e propria inclinazione traduttiva che caratterizzava - a suo dire - l'emergente cultura nazionale di una nazione non ancora esistente.

Giunto a Roma, come tutti i viaggiatori provenienti dal nord, attraverso la Porta Flaminia il 18 novembre 1755, solo dopo svariati mesi Winckelmann decide di stendere i suoi messaggi in italiano. L'uso della lingua equivale alla decisione di prolungare il soggiorno e quindi di entrare in contatto più stretto con la realtà del luogo. In una lettera a Berendis di inizio febbraio 1758 annuncia di avere intanto anche cambiato d'abito indossando le vesti di abate: "Da Ottobre mi vesto da abate, la sola differenza è un nastro azzurro con un orlino bianco applicato a una fascia nera e una cappa nera che non è più lunga della giub-

3 Sul valore delle lettere all'interno dell'opera di Winckelmann cfr. M. Fancelli, Lettere preromane di Johann Joachim Winckelmann, in "Studi Germanici" 2010, pp. 76-77.

4 G. Folena, L'italiano in Europa. Esperienze linguistiche del Settecento, Einaudi, Torino 1983, p. 434.

5 In realtà, pur avendo escluso dal Deutsches Wörterbuch molti lemmi derivanti da altre lingue, la posizione dei Grimm non era banalmente purista. Sull'argomento cfr. U. Fratzke, Zum Fremdwort im Deutschen Wörterbuch, in Das Grimmsche Wörterbuch.Untersuchungen zur lexikographischen Methodologie, a cura di J. Dückert, Leipzig 1987, p. 157. 
ba. I pantaloni sono di velluto". ${ }^{6}$ Poco alla volta il prussiano Winckelmann cerca di divenire romano. ${ }^{7} \mathrm{Dal} 1758$ italianizza il proprio nome, firmandosi nelle lettere agli amici Gio. Winckelmann. Sembrerebbe davvero che a profilarsi sia il desiderio di una nuova identità linguistica. Le lettere in italiano inizia a scriverle quando si sente più padrone di una lingua appresa in parte nel corso degli studi a Jena e poi come autodidatta. ${ }^{8}$ E' perciò poco probabile quanto lui stesso aveva sostenuto in una lettera in latino del 2.4.1747. Allora, ancora prima di arrivare in Italia, ma sempre attento ad esporre le proprie attinenze culturali, aveva affermato di parlare francese e italiano: "conosco la lingua francese e quella degli Italiani e non manco certo familiarità con l'idioma degli Inglesi". In realtà, anche venendo in Italia, la conoscenza dell'italiano rimaneva legata alla lingua scritta, frequentata attraverso la lettura di classici come Dante, Petrarca, Ariosto o Tasso, Guarini, di cui legge il Pastor Fido, e Metastasio, ai quali nel tempo si aggiungono alcuni scrittori contemporanei come Alessandro Guida, Antonio Conti o Vincenzo Gravina. Ma scrivere in italiano è altra cosa. D'altronde è necessario utilizzare la lingua italiana proprio per i messaggi scritti, per tenere per esempio i contatti con la cerchia italiana di Dresda, cioè con Bianconi, nonché per intrattenere rapporti con editori, antiquari e studiosi italiani. La lingua doveva servirgli come strumento di integrazione sia in Italia che fuori, dimostrare l'attenzione data all'interlocutore, mediare la propria identità di straniero.

Come è noto l'italiano si era imposto in Germania per l'area semantica della musica ma anche, grazie non in ultimo a Winckelmann, si affermerà nella sfera dell'arte, ancora tutta da forgiare. Se nei Gedanken über die Nachabmung der griechischen Werke in der Malerei und in der Bildhauerkunst (1755) abbondano i termini francesi (come Contour, Draperie, Ordonance, Colorit), con la venuta in Italia la lingua italiana si afferma quale referente imprescindibile per il linguaggio del classicismo tedesco: il nuovo campo della ricerca artistica necessita un proprio linguaggio che riempie vuoti linguistici dovuti

6 Lett. I, p. 428.

7 Il riferimento a un "römisch gewordenen Preussen" è usato da Winckelmann in una lettera a Gessner del 14 novembre 1761 (Lett. II, p. 266).

8 Cfr. Horst Rüdiger, Winckelmann und Italien: Sprache, Dichtung, Menschen, Krefeld 1956.

9 Lett. I, p. 133. 
a una materia ancora da costruire soprattutto se, come nel volere di Winckelmann, la storia dell'arte dovrà fondarsi sull'attenzione verso gli oggetti d'arte, i materiali e i manufatti. Termini come Loggia, Foro, museo, patina, giallo verde e rosso antico, cubo, tessera, Patera, Villa, arena, bassorilievo e, ancora, intaglio, quadriga, biga, lava, scena, incuneati nei testi tedeschi, rappresentano una tendenza linguistica che farà strada. ${ }^{10}$ Se è ormai da tempo acquisito che la famosa endiadi di edle Einfalt e stille Größe provenga da una traduzione dal francese, altre parole non vengono tradotte, ma direttamente assimilate. Ciò avviene, come ha sottolineato Elisabeth Décultot, ${ }^{11}$ non tanto perché si constata l'intraducibilità dei lemmi, ma perché attraverso il vocabolo straniero, esibito marcatamente come tale, si rinvia a una tradizione culturale impiantando nello stesso tempo quella tradizione nella propria lingua. D'altronde al momento di scrivere la Storia dell'arte Winckelmann si ripromette di "non dire con due parole, ciò che può accadere con una", il che, in ossequio all'ideale della brevitas classica, equivale a preferire il termine tecnico a qualsiasi parafrasi nostrana. ${ }^{12}$ Come nell'unicità del gesto Winckelmann esalta il compassato rigore dell'arte antica, anche la lingua non deve gesticolare a vuoto, disperdendosi nei rivoli di inutili digressioni e ornamenti.

Torniamo all'epistolario. Già Goethe aveva indicato l'importanza delle lettere per comprendere l'identità dell'uomo e dell'autore Winckelmann a cui nel 1805 dedica una biografia pubblicando anche una piccola raccolta di ventisette lettere a Hyeronimus Dietrich Berendis (1719-1782). Per quel grande greco a Roma, come Winckelmann si definì e Goethe amò ricordare, le lettere indicano l'interazione esistente fra la vita e le opere: le lettere sono una sorta di dialogo con se stessi che costituisce un eredità preziosa, uno dei "monumenti più importanti che il sin-

10 Sulla creazione di una terminologia specialistica attinente il campo dell'arte mi permetto di rinviare ad un mio studio uscito in tedesco Aspekte der Sprachvariation bei Winckelmann in "Mitteilungen der Winckelmann Gesellschaft" 68, 2005, pp. 1-7 e in italiano: Interferenze e plurilinguismo nel Settecento: il caso di Winckelmann, in TranScrizioni. Percorsi interculturali nella letteratura e nella lingua tedesca, Annali Sezione Germanica. Istituto Universitario Orientale di Napoli 17 (2007), pp. 571-584.

11 Cfr. E. Décultot, Untersuchungen zu Winckelmanns Exzerptheften. Ein Beitrag zur Genealogie der Kunstgeschichte im 18. Jahrbundert, Ruhpolding 2004, pp. 180 e sgg.

12 Cit. in M. Disselkamp, Die Stadt des Gelehrten. Studie zu Johann Joachim Winckelmanns Briefen aus Rom, Tübingen, 1993, p. 125. 
golo uomo può lasciare". ${ }^{13} \mathrm{Nel}$ caso di Winckelmann le lettere costituiscono fra l'altro le uniche fonti dirette sulla vita romana. Una ricostruzione non semplice perché l'intero carteggio è modulato - come si è detto in base a circostanze del momento e a inquietudini estemporanee. Anche in questo caso Winckelmann appare come il perfetto esempio di un secolo in cui la corrispondenza epistolare rappresenta la fonte primaria di una comunicazione che sostituisce la scrittura all'oralità. Strumento di mediazione, che si colloca a metà fra monologo e dialogo, il genere della lettera è in grado di supplire all'assenza della persona attraverso la presenza della pagina scritta. Inoltre la pratica epistolare è un gesto emancipatorio, appartenente alla diversità di funzioni che attende l'uomo sociale del Settecento sia nella sfera privata che in quella pubblica, visto fra l'altro che proprio adesso quelle due sfere, separate per secoli, trovavano per la prima volta una loro convergenza. Fautore di un moderno stile epistolare fu in Germania Christian Fürchtegott Gellert, professore di morale, poesia ed eloquenza a Lipsia che al suo manuale del 1751 accluse una serie di esempi con la funzione di mostrare la varietà delle diverse situazioni comunicative a cui lo scrivente doveva conformarsi. In un'esplicita equiparazione fra discorso epistolare e corpo umano, l'ideale di semplicità e naturalezza, si contrapponeva in Gellert alla ricercatezza barocca che caratterizza la galanteria cortese, sostenendo l'etica di un gusto lineare, senza decorativismi, in grado di assecondare le esigenze della emergente socialità borghese. Il maestro di retorica educava a utilizzare nell'ambito della scrittura interpersonale la libertà e la leggerezza della conversazione. ${ }^{14} \mathrm{Ma}$ Winckelmann va oltre: la sua libertà è totale, la disinvoltura della lingua parlata si trasforma in noncuranza delle regole e nei toni irruenti della polemica. ${ }^{15}$ D'altronde anche negli scritti in tedesco non si cura tanto di regole grammaticali o ortografiche, la qual cosa gli venne per lungo tempo rimproverata. Sempre August Wilhelm Schlegel

13 J.W. Goethe, Winckelmann und sein Jabrbundert, in Ästhetische Schriften. Sämtliche Werke, Briefe, Tagebücher, a cura di F. Apel, vol. 19, Frankfurt am Main 1998, p. 13.

14 Cfr. C.F. Gellert, Briefe, nebst einer praktischen Abhandlung von dem guten Geschmacke in Briefen, in Gesammelte Schriften, a cura di B. Witte et al., Berlin et al. 1989.

15 Sul tema dello stile polemico di Winckelmann cfr. E. Osterkamp, J.J. Winckelmann "Heftigkeit im Reden und Richten". Zur Funktion der Polemik im Leben und Werk des Archäologen", Winckelmann Gesellschaft, Stendal 1995. Osterkamp sottolinea come l'inclinazione verso la violenza verbale sia connaturata all'urgenza di affermare il nuovo modello del suo metodo storico. 
nel recensire nel 1811 l'edizione delle opere pubblicate a Weimar, pure mettendo in risalto come Winckelmann fosse uno dei pochi scrittori tedeschi di fama europea con uno stile efficace perché allo stesso tempo calmo ed entusiasta, doveva altresì suggerire di emendare e correggere $i$ suoi scritti, ignari di ogni regolarità non solo nell'ortografia (cosa a quel tempo ancora assai diffusa), ma anche nella grammatica, viste le tante improprietà nelle flessioni, nelle scelte lessicali inadeguate, nell'ordine delle parole definito goffo, e addirittura nell'uso errato dei casi. ${ }^{16}$ Inadempienze attribuite da Schlegel a varietà diatopiche con cui Winckelmann si era confrontato fin dalla nascita: l'origine nella regione del Brandeburgo, l'assidua frequentazione delle lingue antiche e, infine, il lungo soggiorno in una terra straniera che gli aveva impedito un contatto diretto con la lingua madre. In realtà, come poi è stato riconosciuto, il mancato rispetto delle norme linguistiche di Winckelmann - cosa che si ripropone anche nell'uso dell'italiano - corrisponde a una precisa esigenza espressiva. L'irregolarità del tedesco è la cifra stilistica di una creatività linguistica, frutto a sua volta della autonomia di pensiero: l'uso di un'interpunzione più ritmica che sintattica è sintomo di una sensibilità linguistica che non può e non deve essere limitata da alcuna normatività.

A Christian Felix Weisse, curatore della rivista Bibliothek der schönen Wissenschaften und der freyen Künste, che gli aveva rivolto l'invito a tradurre uno testo (che si rivelerà essere di Mengs) scrive: "perché in realtà non sono così saldo nelle regole linguistiche e se continuerò a vivere a Roma sarò costretto a rinunciare definitivamente a scrivere in tedesco. Non ho testi tedeschi da leggere, né tantomeno ho la possibilità di parlar bene, e con il mio ciarpame mi si catalogherebbe tra

16 A.W. Schlegel recensisce le opere di Winckelmann pubblicate a Weimar per la cura di Ludwig Fernow, Heinrich Meyer e Johann Schulze. I curatori non avevano normativizzato la lingua di Winckelmann, cosa che Schlegel non condivide: A.W. Schlegel. Sämmtliche Werke, a cura di E. Böcking, vol. XII, Leipzig 1846-47, pp. 321-83 in particolare p. 326. Sulla recensione di Schlegel cfr. il commento al vol. Frühklassizismus. Position und Opposition: Winkelmann, Mengs, Heinse, a cura di H. Pfotenhauer et al., Frankfurt am Main 1995, pp. 345 e sgg.. Alcune interessanti annotazioni sulla lingua e l'ortografia si trovano anche in J.J. Wincklemann, Herkulanische Schriften I Sendschreiben von den Herculanischen Entdeckungen, a cura di Stephanie-Gerrit Bruer und Max Kunze, Mainz 1997, p. 60. Nel volume di H. Zeller, Winckelmanns Beschreibung des Apollo im Belvedere, Zürich 1955, che rivolge particolare attenzione alla lingua e allo stile di Winckelmann, le particolarità ortografiche vengono registrate nel contesto di un atteggiamento ribelle contro ogni forma di pedanteria. Cfr. p. 41. 
i corruttori della lingua" ${ }^{17}$ Se è solo per una captatio benevolentiae che Winckelmann diventa improvvisamente autocritico, anche qui, come del resto nel giudizio di Schlegel, viene suggerito un argomento utile a comprendere la lingua di Winckelmann: il lungo soggiorno in un paese straniero aveva dato vita a un tedesco venato di influssi esterni, destinato ad avere come esito un idioletto, che nasceva dal confronto con la diversità.

Si è detto dell'uso dell'italiano funzionale all'inserimento a Roma in un mondo che non dovette risultare facile per quell'uomo venuto dal nord: basti solo pensare alla realtà di potere legata al clero, con annessi intrighi e rivalità. In Italia, ancora più che le tracce dell'antichità e del suo passato, il figlio del calzolaio di Stendal cercava in primo luogo la libertà che gli permettesse di dedicarsi alla vitale passione per gli studi. Andare - e poi rimanere - in Italia era necessario soprattutto per ragioni finanziarie tanto più che con lo scoppio della guerra dei Sette anni le possibilità di ricevere un sostegno da parte del re di Sassonia, grazie al quale aveva intrapreso il suo viaggio a Roma, erano divenute assai scarse, per non dire nulle. Il suo sostenitore a Dresda era stato il cardinale Archinto che lo aveva aiutato nei primi contatti in Italia. Non prima però che Winckelmann decidesse di abbracciare la fede cattolica, evidente concessione alle aspettative dell'ambiente romano da cui sperava di ottenere sostegno.

Anche se i riconoscimenti gli giunsero, gli anni in Italia, trascorsi soprattutto a Roma, non furono semplici. Il compromesso in materia religiosa è il segno di una contraddizione latente: per diventare autore nel senso moderno della parola, cioè libero nella propria attività, occorreva conformarsi a norme comportamentali e a gerarchie sociali, diverse da quelle della corte sassone, comunque più complesse da decodificare per uno straniero. Da Firenze, dove trascorre più di un anno per redigere il catalogo Stosch, scrive a Bianconi: "Io sto adesso ruminando col mio cervello se ho da comparire in scena fra' Letterati e Antiq. d'Italia o se tornerebbe meglio starmene cheto: io sono piu per il Si che per il NO" ${ }^{18} \mathrm{E}$ come acconsentì alla conversione, così avvenne per l'italiano. Se l'obiettivo era di emanciparsi dando dignità autoriale alla propria attività di scrittore,

17 Lett. II, p. 222. Sullo scritto di Mengs e sull'influenza di Winckelmann cfr. Lett. II, p. 560.

18 Lett., I, p. 547. 
l'italiano era con ogni evidenza uno strumento di negoziazione. E' perciò che fin dall'inizio nelle lettere si parla dell'intenzione di tradurre in italiano la Storia dell'arte dell'antichità, il suo grande progetto romano. Pure se ciò non avvenne $\mathrm{e} \mathrm{W}$. scriverà in italiano solo la sua ultima opera, i Monumenti antichi inediti, prodotta con l'esplicita finalità di inserirsi nel mondo italiano, l'uso della lingua va di pari passo con le difficoltà di un'integrazione sociale che, nonostante le diverse attestazioni nel carteggio, non avvenne mai stabilmente. ${ }^{19}$ Seppure in una lettera del 1762 all'amico e musicista Marpurg Winckelmann dipinge con soddisfazione quanto ha raggiunto a Roma, le cose non dovevano stare proprio in questi termini o almeno non si può dare alle sue parole unilaterale e definitiva certezza: "Io direi: ho vissuto otto anni. Questo periodo corrisponde al mio soggiorno a Roma e in altre città d'Italia. Qui ho cercato di richiamare in vita la mia gioventù, che ho perduto in parte nel lavoro, in parte nelle preoccupazioni, e almeno morirò potessi pensare, sperare e guadagnare". ${ }^{20}$

In verità la vita nella comunità romana risultò a Winckelmann assai più problematica di quanto queste righe possano dare a intendere. Anche a Roma il suo impegno fu spesso rivolto a trovare un modo per finanziare i propri studi e anche da quando divenne consulente del Cardinale Albani, la paga di 26 scudi che questi gli corrispondeva mensilmente non doveva essere sufficiente, ${ }^{21}$ se comunque si adoperò per trovare altre fonti di sostentamento come quella di scriptor della lingua teutonica presso la Biblioteca vaticana a cui si aggiungerà la nomina di Sovrintendente delle Antichità di Roma.

La battaglia portata avanti in vista della libertà di pensiero era destinata a trovare non pochi ostacoli: nelle invidie degli studiosi (che non vedevano di buon occhio l'avanzare di uno straniero nella considerazione degli intenditori d'arte), ma anche nelle riserve ideologiche di

19 Per l'italiano dei Monumenti antichi inediti cfr. l'intr. di Maria Fancelli e di al volume nell'edizioni degli Schriften und Nachlaß, uscita presso l'editore von Zabern di Mainz, 1999 e sgg.. Qui si fa il punto sulla conoscenza della lingua italiana da parte di Winckelmann. Per quanto riguarda l'opera stesa in italiano, al cui progetto il fondatore della Storia dell'arte attribuì grande importanza, l'assenza di manoscritti non permette di indicare con sicurezza l'apporto dei revisori, di cui il saggio dà per la prima volta notizia in maniera esauriente.

20 Lett. II, p. 370.

21 C. Justi, Sein Leben, seine Werke und seine Zeitgenossen, vol. 2, Winckelmann in Italien, Leipzig 1872, p. 26. 
fronte all'innovazione di una storia dell'arte antica ricostruita in base alle testimonianze visive. La novità del pensiero di Winckelmann agiva in contrasto con antiquari e studiosi italiani, ritenuti spesso, letteralmente, "asini" e "imbrattacarte", privi di ogni dignità scientifica o letteraria. Senza colpo ferire Winckelmann trasferiva la sua verve polemica da una lingua all'altra con conseguente arricchimento dell'italiano sull'onda di risonanze tedesche. Uno scambio che avviene in realtà a diversi livelli. La lingua delle lettere è costellata da interferenze di vario tipo: le frasi infinitive, risentendo del costrutto tedesco, danno vita a frasi del tipo "io ero già risoluto di vendicarmi", "mi ha invitato di godere", "non è possibile a figurarsi" "i miei amici mi sono esibiti di farmi ottenere"), l'uso degli articoli e delle preposizioni articolate, con relativa mancata individuazione del genere di appartenenza, derivano dalle differenze con la lingua madre da cui si riprende la costruzione dei verbi funzionali ("ans Licht treten") o la collocazione dell'aggettivo anteposto al sostantivo. A quelle tedesche si aggiungono poi le interferenze dal latino, evidenti in parole come benedittione, afflittione aspettatione, scritte raddoppiando la dentale t. Alle interferenze si affiancano le oscillazioni ortografiche, gli scambi fonetici (tipica è quello fra la fricativa alveolare sorda e sonora di pranzo e pranso), la difficoltà nelle consonanti doppie che produce fenomeni di degeminazione o di ipergeminazione, il passato remoto preferito assai spesso al passato prossimo. Contemporaneamente però, accanto alla natura ibrida dell'italiano di Winckelmann, si scorge una capacità di penetrazione di una lingua di cui il prussiano divenuto romano, come lui stesso si definì, doveva avvertire col tempo l'identità: "Vuoi sapere che lingua parlo" scrive all'amico Berendis nel 1758. "Che altro se non italiano. Ma il molto studio e la scarsa vita sociale mi hanno molto frenato. Questa lingua è più difficile di quanto si pensi leggendo i libri. E' ricca come il greco e l'accento romano s'impara difficilmente. Ma visto che parlo con Principi e Cardinali, puoi ben capire che so il necessario". ${ }^{22}$ La coscienza del suo italiano imperfetto era quindi assolutamente presente se ancora nello stesso anno in una lettera a Bianconi scriveva: "Scusi li sgorbi nello scrivere in riguardo del poco tempo che ci posso mettere. Dello stilo non parlo: è vizioso, lo so: ma la benedetta Lingua Italiana facile in apparenza \& quae se offert discentibus - ut sibi quivis Speret idem) - piu che

22 Lett. I p. 430. 
si crede d'avanzare, più difficile riesce" ${ }^{23}$ Con tutti i limiti del caso, cioè con tutta la coscienza dei propri limiti linguistici, Winckelman sente la vitalità dell'idioma straniero. Del resto anche per le opere d'arte il suo metodo reclamava in luogo dell'erudizione libresca la visione diretta, l'unica che garantisce la precisazione del dettaglio, da cui non in ultimo deriva la contestualizzazione storica dello stile. E lì dove si tratta invece di tornare ai libri e di leggere e copiare (lo studioso di antichità ne ha fatto ampia esperienza attraverso le trascrizioni e gli appunti delle sue letture), ciò non avverrà più nel senso di una attestazione di cultura e di sapere. Leggere e copiare significa ricreare. Del resto chi meglio di lui poteva sapere che si diventa originali per imitazione? E così prende spazio un linguaggio metaforico denso e articolato: "mi sono ingolfato in un mare dove non mi mancherà per lungo tempo acqua da navigare", scrive parlando del lavoro che sta svolgendo a Firenze per catalogare la collezione di gemme del barone Stosch. ${ }^{24}$ Anche l'eccesso reverenziale può rivestirsi di un'espressività metaforica, come avviene nella stessa lettera: "Ella fa come gia fece Iddio con Mosè, sollevandomi fin'all'altissima cima della mia speranza da dove quantunque in vista rimota mi mostra da una parte la terra di promissione, dall'altra la Grecia e l'Ionia". ${ }^{25}$ Il polemista Winckelmann fa sentire i suoi strali anche in terra italiana. Il desiderio di appropriazione si traduce in un impeto pressoché furioso di conquista ("V. S. Ill. sappia ancora ch'io studio arrabbiatamente la Lingua italiana quanto mi permette La Lettura Greca”, scrive sempre a Bianconi il 17 aprile 1757). ${ }^{26}$ Sulla scia del sistema derivativo dell'italiano dilatato per analogia (ne sono esempio parole come "esaltamento", "assegnamento", "indiscretezza", "inquietezza") vengono create parole che risentono da un lato della prolificità morfologica e lessicale dei composti tedeschi e dall'altro dell'ampiezza dei suffissi italiani, assai più limitati in tedesco, che caratterizzano l'ambito degli accrescitivi o dei peggiorativi. Nascono estensioni ridondanti come prolississimo, arcisolito, più che arcicontento, nuova arcinuova, lì dove la ricorrenza del prefisso arci è evidentemente frutto di analogia con il prefisso tedesco rafforzativo - e con funzione generalmente peg-

\footnotetext{
23 Lett. I, p. 485.

24 Lett. I, p. 540.

25 Ivi, p. 539.

26 Ivi, p. 367.
} 
giorativa - Erz (derivante a sua volta dal latino antico nonché dal greco), usato da Winckelmann in parole come Erzpedant. Ma il sistema dell'estensione diviene ancora più espressivo quando l'autore vuole costruire invettive calzanti: appella come Disegnatoraccio il direttore del museo di Portici Camillo Paderni, descrivendo poi apertamente la sua disistima verso gli esperti napoletani: "Ho contraffatto il sempliciotto più che non lo sono per non risvegliare la gelosia degl'Ignoranti a cui e confidata l'Intendenza dell'Antichità". ${ }^{27}$ Parla di "Semidottoracci" ${ }^{28}$ a proposito degli esperti di lingue antiche della vaticana, dei Francesi scioli (dalla voce latina sciolus semidotto) che cinguettano dell'arte, di "Antiquarucoli" e "Florentacci" o, prendendo sempre di mira gli antiquari romani, di "Scartabellatori di vocaboli e bagattellacce". La lingua, si potrebbe dire, si arricchisce per via esogena sfruttando le proprie possibilità endogene.

Se Winckelmann parlava con violenza e non come un letterato erudito, è perché sentiva l'urgenza di affermare il proprio messaggio e con esso la nuova identità di autore: la polemica di cui era fautore, dava vita a una lingua diretta e violenta. Contro uno scritto pubblicato in risposta alle critiche avanzate a proposito delle scoperte di Ercolano, opera di Bernardo Galiani e Matteo Zarillo, Winckelmann non lenisce i modi e scrive a Mengs ( 8 nov. 1765): "Le invettive somaresche del Libello ingiurioso han fatto poco colpo sopra di me, vedendo che questa Charta cacata rimane aborrita da' Napolitani medesimi". ${ }^{29}$ Insomma: il polemista che, citando Catullo, attacca il mondo dell'erudizione e combatte la pedanteria e l'ignoranza degli antiquari, sfrutta la conoscenza della lingua italiana per dire oltre ogni lingua. E così l'idioma musicale per eccellenza che si parla nelle corti di tutta Europa acquisisce nel furore degli attacchi verbali di Winckelmann la pregnanza di una lingua impetuosa, canzonatoria, adatta a invettive che il prussiano non divenuto mai romano, l'amante della calma classica, seppe forgiare a emblema del suo credo nella verità delle idee.

27 Ivi, p. 456.

28 Ivi, p. 420.

29 Lett. III, p. 182. 